\title{
Austrocknung von Bächen - eine Gefahr für die Wasserqualität?
}

\author{
Gabriele Weigelhofer (D) Michael Tritthart $(D$
}

Online publiziert: 29. April 2019

(C) Der/die Autor(en) 2019

\begin{abstract}
Zusammenfassung Wasserknappheit stellt eine der größten Herausforderungen für das Gewässermanagement im 21. Jahrhundert dar. Bereits jetzt zählt ein Großteil des weltweiten Gewässernetzes $\mathrm{zu}$ den intermittierenden, also phasenweise austrocknenden, Fließgewässern. Durch die fortschreitende globale Erwärmung werden Fließgewässer zunehmend auch in gemäßigten Klimazonen wie Österreich über längere Perioden trockenfallen. Das Projekt PURIFY (2018-21) hat zum Ziel, die Auswirkungen von Austrocknung auf die Wasserqualität und die Selbstreinigungskapazität von Bächen in verschiedenen Regionen Österreichs zu untersuchen und Faktoren zu identifizieren, die sich positiv auf die Widerstandsfähigkeit gegenüber Austrocknung auswirken. Zu diesem Zweck wurden 2018 fünf perennierende und fünf intermittierende Bachabschnitte im Burgenland, in der Steiermark und in Kärnten vor und während der Austrocknung bezüglich der Wasserqualität und der Aktivität sedimentgebundener

sich keine signifikanten Unterschiede in der mikrobiellen Atmung, der Bakterienanzahl und den Enzymaktivitäten zwischen perennierenden und intermittierenden Abschnitten derselben Region. Wir nehmen an, dass eine starke Beschattung durch Ufergehölze sowie ein hoher Feinsedimentanteil dabei helfen, eine hohe Restfeuchte in den Sedimenten zu erhalten und somit die Wirkung von Austrocknung auf mikrobielle Prozesse zu reduzieren. Allerdings führten die abnehmenden Durchflüsse sowohl in intermittierenden als auch in perennierenden Bereichen zu starken Schwankungen in den Nährstoffkonzentrationen. Erweiterte Freilanduntersuchungen im Jahr 2019, in Kombination mit Laborexperimenten und hydrodynamisch-numerischen Modellierungen, sollen klären, wie sich periodische Austrocknung kurz- und langfristig auf die mikrobielle Stoffaufnahme im Gewässer und die Wasserqualität in Abhängigkeit vom Gewässertyp und der stofflichen Belastung auswirkt.
\end{abstract} Mikroorganismen analysiert. Trotz der ausgedehnten Trockenphase zeigten

\footnotetext{
Mag. Dr. G. Weigelhofer

Department für

Wasser-Atmosphäre-Umwelt, Institut für Hydrobiologie und Gewässermanagement, Universität für Bodenkultur Wien, Gregor Mendel-Straße 33, 1180 Wien, Österreich

WasserCluster Lunz,

Dr.-Carl-Kupelwieser-

Promenade 5, 3293 Lunz/See, Österreich

gabriele.weigelhofer@wcl.ac.at

PD DI Dr. M. Tritthart

Department für

Wasser-Atmosphäre-Umwelt, Institut für Wasserbau, Hydraulik und Fließgewässerforschung, Universität für Bodenkultur Wien, Muthgasse 107, 1190 Wien, Österreich michael.tritthart@boku.ac.at
}

Schlüsselwörter Klimawandel . Austrocknung · Fließgewässer . Wasserqualität · Selbstreinigung . Mikrobielle Aktivität

\section{Intermittency of streams - a risk} for water quality?

Abstract Water scarcity is one of the
greatest challenges for stream man-
agement in the 21 st century. Already
now, the majority of channel lengths
of natural waterways are intermittent
worldwide. Global warming will in-
creasingly cause perennial streams to
become intermittent also in temperate
regions such as Austria. The project
PURIFY (2018-21) aims at investigating
the impacts of intermittency on the
water quality and the self-purification
capacity of streams in different regions
in Austria. We want to identify fac-
tors, which significantly influence the
resistance of in-stream processes to- wards desiccation. For this purpose, we sampled five perennial and five intermittent streams in Burgenland, Styria and Carinthia before and during the dry phase in 2018 to analyse the water quality and the activity of benthic microorganisms. Despite an extended dry period, we observed no significant differences in the microbial respiration, abundances, and enzymatic activities between perennial and intermittent reaches within the same region. We assume that dense shading by riparian trees and high accumulations of fine particles in the sediments helped to maintain a high humidity in the sediments and, thus, buffered the effects of drying on microbial processes. However, receding water levels in both intermittent and perennial reaches resulted in large fluctuations of nutrient concentrations. Extended field investigations combined with laboratory experiments and hydrodynamic modelling will clarify the short- and longterm effects of repeated drying on the microbial uptake of nutrients (self-purification capacity) and on the water quality in relation to stream type and degree of pollution.

Keywords Climate change · Intermittency $\cdot$ Streams $\cdot$ Water quality $\cdot$ Self-purification $\cdot$ Microbial activity

\section{Einleitung}

Ausgedehnte Dürreperioden und die daraus resultierende Wasserknappheit stellen eine der größten Herausforderungen für das Gewässermanagement im 21. Jahrhundert dar (Whitehead et al. 2009). Während der letzten 30 Jahre stieg die Häufigkeit und Dauer von Trockenperioden in Europa deutlich an (IPCC 2007; Sutherland et al. 2008; Wilby et al. 2006). Geringe Niederschlagsintensitäten im Sommer und Winter sowie die Beeinträchtigung des Wasserrückhalts im Einzugsgebiet durch die Verbauung offener Flächen führen 


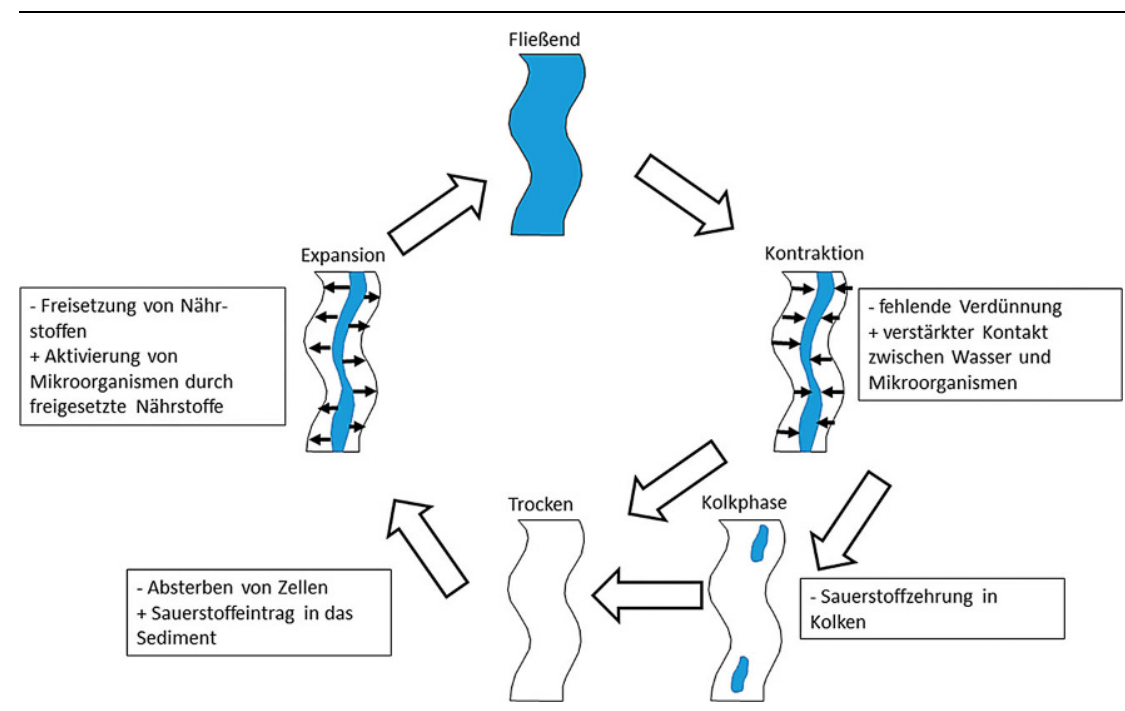

Abb. 1 Verschiedene Phasen der Austrocknung von Gewässern und mögliche positive (+) und negative (-) Folgen für die Wasserqualität. (Modifiziert nach Romani et al. (2017) und Von Schiller et al. (2017))

zu Rückgängen des Wasserdargebots in Grund- und Oberflächenwasserkörpern. Bereits jetzt zählen über $50 \%$ des weltweiten Gewässernetzes zu den phasenweise austrocknenden (intermittierenden) oder phasenweise wasserführenden (ephemeren) Fließgewässern, wobei dieser Anteil aufgrund der fehlenden hydrologischen Informationen über Gewässeroberläufe voraussichtlich stark unterschätzt ist (Datry et al. 2014, 2017). Im Zuge der fortschreitenden globalen Erwärmung und Klimaveränderung ist $\mathrm{zu}$ erwarten, dass Fließgewässer zunehmend auch in gemäßigten Klimaregionen wie Österreich über längere Perioden trockenfallen werden, die ursprünglich das ganze Jahr über Wasser führten (perennierend).

Bei der Austrocknung von Fließgewässern wechseln einander verschiedene hydrologische Phasen ab (Romani et al. 2017; Von Schiller et al. 2017; Abb. 1). Während der Kontraktion kommt es zunächst zu einer deutlich sichtbaren Reduktion der benetzten Oberfläche im Bachbett. In der Kolkphase ist kein zusammenhängender oberirdischer Abfluss mehr erkennbar, tiefere Stellen im Bach sind noch mit Wasser gefüllt. In der Trockenphase ist schließlich das gesamte Bachbett oberirdisch trocken. Je nach den vorherrschenden Umweltbedingungen (u.a. geologischer Untergrund, Hydrologie und Klima) können trockenfallende Bäche alle oder auch nur einzelne Phasen durchlaufen. Ebenso können sich Kontraktion, Kolkphase, Trockenphase und
Expansion während einer Trockenperiode mehrmals abwechseln, bevor der Bach wieder in eine längere Fließphase übergeht.

Die Austrocknung von Bächen wirkt sich nicht nur auf deren sichtbare Lebewelt aus, sondern beeinflusst auch die mikrobiellen Gemeinschaften in den Sedimenten, die für die Stoffaufnahme und damit für die Selbstreinigung in den Gewässern zuständig sind (Von Schiller et al. 2017). Diese Selbstreinigung beschreibt die Fähigkeit von Fließgewässern, eingetragene Schmutz- und Schadstoffe aus dem Umland durch abiotische und biotische Prozesse $\mathrm{zu}$ binden und so möglichst permanent aus dem Wasserkreislauf zu entfernen (McColl 1974; Peterson et al. 2001; Weigelhofer et al. 2018). Austrocknung, aber auch Wiedervernässung kann die involvierten Mikroorganismen aufgrund von osmotischem Stress schädigen und sich - zumindest kurzfristig - negativ auf die Selbstreinigungskapazität auswirken (Sabater et al. 2016; Timoner et al. 2012; Abb. 1). Die Intensität der Schädigung und die Zeit bis zur Erholung des aquatischen Mikrobioms hängen dabei vor allem vom Ausmaß der Austrocknung ab. Eine ausreichende Restfeuchte im Sediment kann zum Beispiel die Wirkung der Austrocknung auf die Mikroorganismen deutlich verringern und die Erholungsdauer reduzieren (Marxsen et al. 2010; Oprei et al. 2019). Daneben spielt auch die jeweilige hydrologische Situation eine wesentliche Rolle für die Wasserqualität. Sowohl vor als auch nach der Trockenphase (Kontraktion und Expansion) kann es aufgrund der fehlenden Verdünnung zu erhöhten Nährstoff- und Kohlenstoffkonzentrationen in der Wassersäule kommen (Delpla et al. 2009; Murdoch et al. 2000; Von Schiller et al. 2017; Abb. 1). Die gleichzeitig erhöhten Wassertemperaturen steigern die mikrobiologische Mineralisation von organischem Material, was zu einer Freisetzung von Nährstoffen aus den Sedimenten führen kann (Whitehead et al. 2009). Andererseits können die reduzierten Strömungsgeschwindigkeiten und die längeren Verweilzeiten auch in einer verstärkten mikrobiellen Aufnahme von Nährstoffen im Bach resultieren (Gomez et al. 2017).

Trotz der zunehmenden Anzahl an Studien über intermittierende Gewässer, vor allem im mediterranen Raum während der letzten 10 bis 15 Jahre (z.B. Bernal et al. 2013; Romaní et al. 2013; Sabater et al. 2016; Von Schiller et al. 2011), ist bis dato wenig darüber bekannt, wie sich Austrocknung auf die Wasserqualität von Bächen in gemäßigten Klimazonen auswirkt und welche Faktoren die Widerstandsfähigkeit der Gewässerselbstreinigung gegenüber Austrocknung erhöhen. Gerade in Hinblick auf eine mögliche weitere Zunahme von intermittierenden Gewässerstrecken ist es für ein nachhaltiges Gewässermanagement notwendig, Konzepte für eine Risikoabschätzung hinsichtlich einer zukünftigen Zustandsverschlechterung durch Austrocknung zu entwickeln. Das Klimafondprojekt PURIFY (2018-21) hat daher zum Ziel, die Auswirkung von Austrocknung auf die Wasserqualität und die Selbstreinigungskapazität von Bächen in verschiedenen Regionen Österreichs systematisch zu untersuchen und Faktoren zu identifizieren, die sich positiv oder negativ auf die Resistenz der Gewässerselbstreinigung gegenüber Austrocknung auswirken. Folgende Teilaspekte werden im Projekt behandelt:

- Analyse der kurz- und mittelfristigen Auswirkungen von Trockenperioden auf die Wasserqualität und die mikrobiellen Prozesse in intermittierenden Bächen im Vergleich zu perennierenden Gewässern mittels Probennahmen im Freiland.

- Experimentelle Untersuchung der unmittelbaren Folgen von Austrocknung und Wiedervernässung auf 


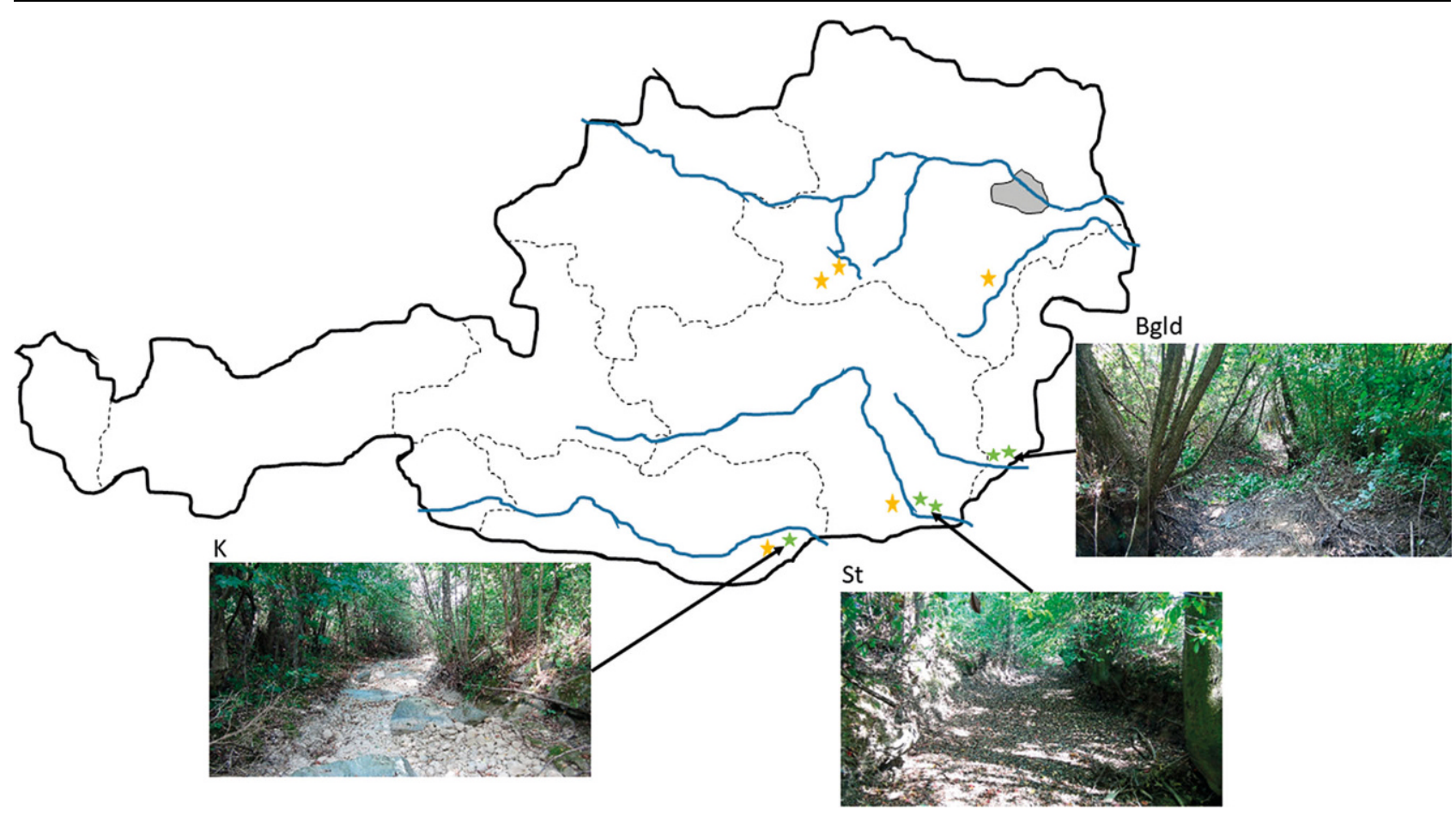

Abb. 2 Lage der Untersuchungsgewässer 2018 (grüne Sterne) und zusätzliche Untersuchungsgewässer 2019 (orange Sterne) in Kärnten $(K)$, Niederösterreich, dem Burgenland $(B g / d)$ und der Steiermark (St). Die Bilder zeigen jene Gewässer, die mit einem numerischen Modell detaillierter untersucht werden

die Selbstreinigung in Gewässersedimenten.

- Identifikation von externen (z.B. Länge und Intensität der Trockenperiode) und internen Faktoren (z. B. Sedimentbeschaffenheit, stoffliche Belastung), die die Resistenz bzw. die Vulnerabilität von Gewässeroberläufen maßgeblich beeinflussen.

- Modellierung der Konsequenzen von zukünftig zu erwartenden Trockenperioden auf die Wasserqualität ausgewählter Gewässeroberläufe.

- Entwicklung eines Leitfadens für eine Risikobewertung hinsichtlich einer zukünftigen Verschlechterung der Wasserqualität.

Durch das Projekt soll die Forschung über intermittierende Gewässer in gemäßigten Klimazonen sowohl in Österreich als auch international gestärkt werden. Im Folgenden werden wesentliche Ergebnisse aus den Freilanduntersuchungen 2018 präsentiert und ein Ausblick auf weitere Untersuchungen gegeben.

\section{Methoden}

Im ersten Projektjahr wurden jeweils vier Bachabschnitte im Burgenland, zwei in Kärnten und vier in der Steier- mark untersucht, wobei die eine Hälfte der Probenstellen in trockenfallenden und die andere Hälfte in permanent wasserführenden Bereichen lag (Abb. 2). In jedem Bundesland wurden die Bäche so ausgewählt, dass sie aufgrund der örtlichen Nähe ähnliche Bedingungen bezüglich geologischem Untergrund, Boden, Hydrologie, Landnutzung und Klima aufwiesen. Alle Strecken zeigten einen mäandrierenden Bachverlauf und wurden beidseitig von Ufergehölz begrenzt. Die Sedimente waren in den burgenländischen und steirischen Bächen durch Feinkies mit einem hohen Anteil an Feinsediment (Ton, Schluff, Sand) und organischem Material charakterisiert. In Kärnten wiesen die Bachabschnitte hauptsächlich grobkiesiges Material auf.

Alle Bachstrecken wurden jeweils einmal vor der Austrocknung Ende Mai/ Anfang Juni sowie dreimal während der Austrocknung Ende Juli/Anfang August, Mitte September und Anfang November 2018 beprobt. Bei jeder Probennahme wurden Wasserproben aus der Fließrinne bzw. in den trockenen Abschnitten aus wasserführenden Kolken entnommen, soweit welche vorhanden waren, und hinsichtlich der Gehalte an Nitrat, Ammonium, Nitrit und gelöstem reaktiven Phosphor (SRP) mit einem
Continuous Flow Analyzer und gelöstem organischen Kohlenstoff (DOC) mit einem Sievers*900 portable TOC-Analyzer untersucht. Zusätzlich wurden je fünf Sedimentproben aus den oberen $5 \mathrm{~cm}$ der Sedimentschichte entnommen und die Fraktion $<4 \mathrm{~mm}$ bezüglich Wassergehalt, Gehalt an organischem Material, Bakterienzahlen, mikrobieller Atmung und der Aktivität der extrazellulären Enzyme analysiert. Der Wassergehalt wurde über die Differenz zwischen Nass- und Trockengewicht ermittelt, der organische Anteil durch Verbrennen bei $450^{\circ} \mathrm{C}$ über vier Stunden (aschefreies Trockengewicht). Die Bakterienanzahl wurde nach Anfärben mit Sybr Green II im Flowcytometer (Cyto-Flex, Beckman-Coulter) bestimmt (Duhamel und Jacquet 2006). Die mikrobielle Atmung gibt Aufschluss über die Aktivität der Mikroorganismen im Sediment. Sie wurde durch DunkelInkubation der Sedimente über vier Stunden bei Raumtemperatur in gasdichten Flaschen ermittelt. Dabei wurden jeweils $10 \mathrm{~g}$ nasses oder trockenes Sediment in die Flaschen eingewogen und diese luftdicht verschlossen. Nach vier Stunden wurden Gasproben aus den Flaschen gezogen und in einem Gasanalyzer Picarro G2201-I hinsichtlich der Zunahme an $\mathrm{CO}_{2}$ gegenüber 


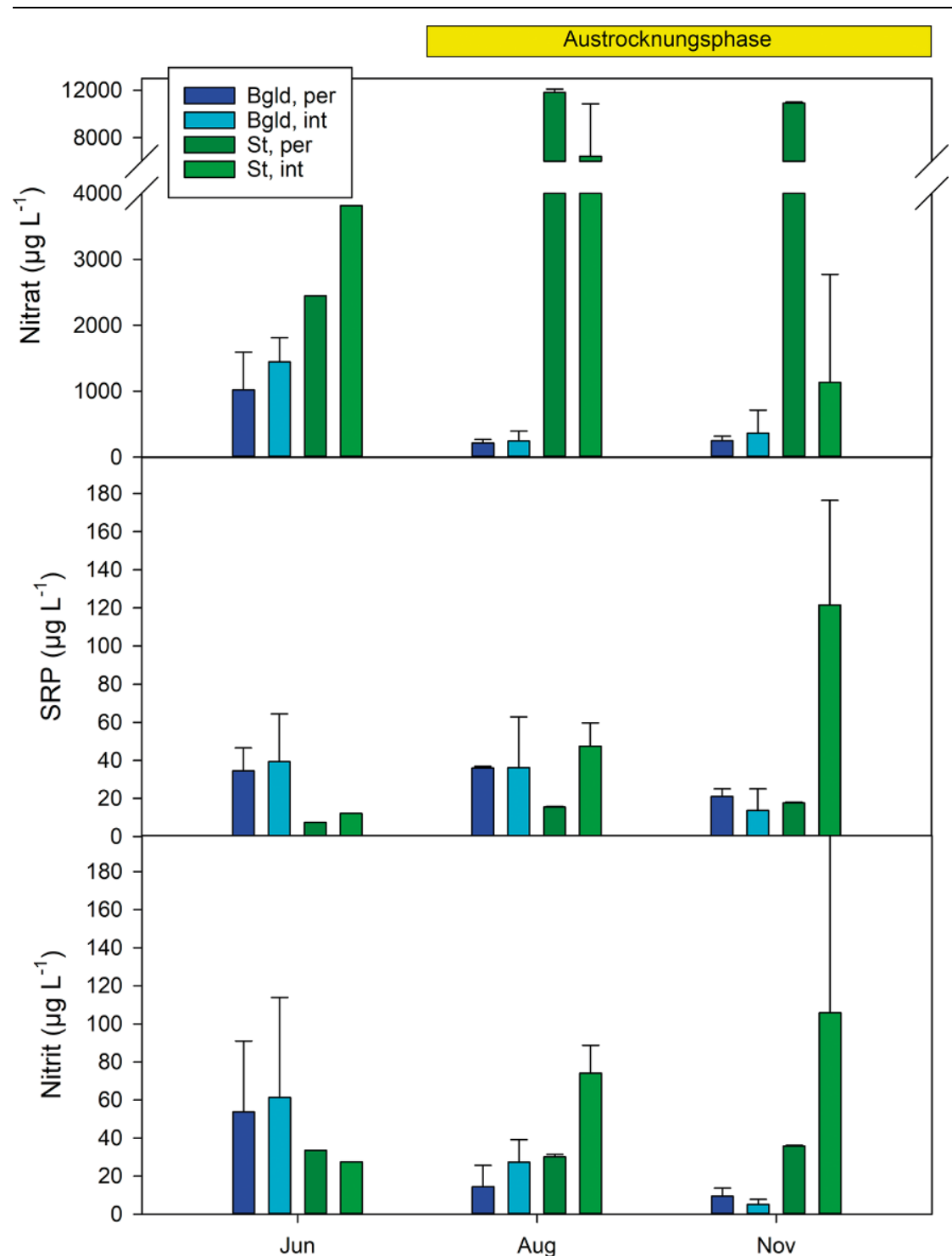

Abb. 3 Nährstoffkonzentrationen in perennierenden (per) und intermittierenden (int) Bachstrecken im Burgenland (Bgld) und in der Steiermark (St; Mittelwerte plus Standardabweichungen, $n=2-4$ ). Der gelbe Balken kennzeichnet die Austrocknungsphase. Daten aus intermittierenden Abschnitten während der Austrocknung stammen von vereinzelten Kolken im Bachbett

dem Anfangswert analysiert. Die Aktivität der extrazellulären Enzyme gibt Aufschluss über die Mineralisation, also den mikrobiellen Abbau von organischen Stoffen, und kann ebenfalls verwendet werden, um Rückschlüsse auf den Zustand und die Aktivität des Mikrobioms im Sediment ziehen zu können. Zu diesem Zweck wurden den Sedimenten künstliche, an eine fluoreszierende Trägermatrix gebundene, Substrate zugefügt und die Substrathydrolyse über die Zunahme der Fluoreszenzintensität wurde in einem 96well Mikroplate Reader (VARIOSCAN
FLASH, Thermo Fisher Scientific) gemessen (Bell et al. 2013).

\section{Erste Ergebnisse und Diskussion}

\subsection{Nährstoffkonzentrationen im} Wasser

In der Abb. 3 sind exemplarisch die Nitrat-, Phosphat- und Nitritkonzentrationen im Wasser an jeweils 2 intermittierenden und 2 perennierenden Gewässerstrecken im Burgenland und in der Steiermark vor und während der Trockenperiode 2018 dargestellt. Anzumerken ist, dass aufgrund der eingeschränkten Anzahl an Probenstellen und der geringen Probenzahl pro Bachabschnitt derzeit keine statistisch gesicherten Aussagen getroffen werden können. Auffallend sind zunächst die relativ starken saisonalen Schwankungen vor allem beim Nitrat, die sich sowohl in trockenfallenden als auch in wasserführenden Abschnitten zeigten. So nahmen die mittleren Nitratkonzentrationen im Burgenland von $1-1,4 \mathrm{mg} \mathrm{L}^{-1}$ im Juni auf $0,2-0,3 \mathrm{mg} \mathrm{L}^{-1}$ im August ab (Abb. 3). In der Steiermark wiederum kam es in den perennierenden Abschnitten zu einem Anstieg von $2,5 \mathrm{mg} \mathrm{L}^{-1}$ im Juni auf $>10 \mathrm{mg} \mathrm{L}^{-1}$ im August und November, während in den intermittierenden Bereichen zunächst ein Anstieg im August, aber danach eine deutliche Reduktion bis November zu beobachten war. Ähnliche Schwankungen zeigten auch die Konzentrationen von gelöstem organischem Kohlenstoff im Wasser. Die mittleren DOC-Gehalte nahmen im Burgenland in den perennierenden Bereichen kontinuierlich von $9 \mathrm{mgL}^{-1}$ im Juni auf $3,2 \mathrm{mgL}^{-1}$ im November $\mathrm{ab}$, in den intermittierenden Bereichen kam es zu einer Abnahme von 9,5 auf $4,4 \mathrm{mgL}^{-1}$. In der Steiermark kam es wie beim Nitrat zu unterschiedlichen Entwicklungen der Konzentrationen in wasserführenden und austrocknenden Abschnitten. In den perennierenden Bereichen nahmen die durchschnittlichen DOC-Konzentrationen von $5,4 \mathrm{mgL}^{-1}$ auf $2,8 \mathrm{mgL}^{-1}$ ab. Hingegen kam es in den intermittierenden Bereichen zu einer Zunahme von $4 \mathrm{mgL}^{-1}$ auf $9,3 \mathrm{mgL}^{-1}$. Ähnlich variable Nährstoffbedingungen in intermittierenden Fließgewässern wurden auch in anderen Studien beobachtet und auf die starken Schwankungen in der Hydrologie dieser Gewässer zurückgeführt (Von Schiller et al. 2011, 2017). In unserer Studie war die variable Wasserchemie nicht nur in den intermittierenden Abschnitten, sondern auch in den perennierenden zu beobachten. Tatsächlich kam es aufgrund der ähnlichen Umweltbedingungen auch an den perennierenden Untersuchungsstellen zu einem deutlichen Rückgang der Wasserführung mit zunehmender Trockenheit (Kontraktion). Ob bei Fortdauer der Trockenperiode ein Anstieg oder eine Abnahme der Nährstoffkonzentrationen $\mathrm{zu}$ beobachten ist, liegt daran, welche physikalischen und biogeochemischen Prozesse im Gewässer dominieren. Eine Abnahme der Konzentrationen weist auf eine verstärkte 




Abb. 4 Mikrobielle Atmung, gemessen anhand der $\mathrm{CO}_{2}$-Produktion bei DunkelInkubation, in nassen und trockenen Sedimenten aus perennierenden (,,fließend") und intermittierenden (,Kolk“, „trocken“) Bachstrecken im Burgenland (Bgld), der Steiermark (St) und Kärnten $(K)$ zwischen Juli und September 2018. Zu sehen sind der Median, die 10-, 25-, 75- und 90-\%-Perzentilen und Ausreißer $(n=5-12)$

Aufnahme und Speicherung der Nährstoffe und des Kohlenstoffs durch das aquatische Mikrobiom hin. Inwieweit diese Aufnahme nur temporär ist oder tatsächlich zu einer Entfernung der Stoffe aus dem System führt (z. B. durch Veratmung von Kohlenstoff oder Denitrifikation von Nitrat zu Luftstickstoff) muss noch untersucht werden. Eine Zunahme der Konzentrationen in der Wassersäule kann hingegen durch die fehlende Verdünnung ebenso wie durch einen verstärkten Abbau von partikulärem organischen Material und die Freisetzung von gelösten Stoffen aus dem Sediment verursacht werden (Gomez et al. 2017; Von Schiller et al. 2017).

Zwar konnten im ersten Projektjahr regionale Unterschiede in den Nährstoffkonzentrationen beobachtet werden, innerhalb derselben Region unterschieden sich perennierende und intermittierende Bachabschnitte vor und zum Teil auch während der Austrocknung jedoch nicht signifikant. Lediglich in einem steirischen Bach kam es während der Trockenphase in Restwasserkolken zu einem Anstieg im Phosphorund Nitritgehalt und einer Reduktion der Nitratkonzentrationen, was auf eine Sauerstoffzehrung in diesen Bereichen hinweist (Romani et al. 2017). Die ersten Ergebnisse lassen somit derzeit keine langzeitigen Auswirkungen der Austrocknung auf die Nährstoffkonzentrationen in intermittierenden Bächen im Vergleich zu perennierenden Bä- chen derselben Region erkennen. Es ist geplant, 2019 detaillierte Freilanduntersuchungen mit einer größeren Anzahl an Probenstellen und Probennahmen in verschiedenen Regionen Österreichs durchzuführen, um diese Frage in Abhängigkeit vom Gewässertyp und der jeweiligen stofflichen Belastung zu klären.

\subsection{Mikrobielle Prozesse im Sediment}

Die mikrobielle Atmung im Sediment zeigte entgegen unseren Erwartungen in den burgenländischen und steirischen Bächen keine signifikanten Unterschiede zwischen perennierenden Bächen und wasserführenden Kolken bzw. trockenen Bereichen in intermittierenden Bächen (Abb. 4). Lediglich im Kärntner Bach wies der ausgetrocknete Abschnitt eine signifikant niedrigere $\mathrm{CO}_{2}$-Produktion auf als der stromaufwärts gelegene wasserführende Abschnitt. Ein Grund dafür könnte die relativ hohe Restfeuchte von 10 bis $15 \%$ Wasseranteil in den Sedimenten der ersten beiden Regionen sein, die sich im feinkörnigen Substrat auch aufgrund der Beschattung der Bäche trotz der langen Austrocknungsphase von Anfang Juni bis Anfang November halten konnte (Marxsen et al. 2010). Im Gegensatz dazu wiesen die trockenen, grobkörnigeren Sedimente im stärker sonnenexponierten Kärntner Bach nur mehr einen Wassergehalt von 1 bis ma- ximal 5\% auf. Die Beschattung durch Ufergehölz scheint somit neben der Sedimentbeschaffenheit ein wesentlicher Faktor für die Resistenz der Mikroorganismen im Sediment gegenüber Austrocknung zu sein.

Die Bakterienzahlen und die Enzymaktivitäten in den Sedimenten untermauern die Ergebnisse der mikrobiellen Atmung zum größten Teil (Tab. 1). Aufgrund der hohen Variabilität konnten keine signifikanten Unterschiede zwischen den perennierenden und den intermittierenden Bachabschnitten innerhalb derselben Region festgestellt werden. Generell ist festzustellen, dass extrazelluläre Enzyme Trockenperioden relativ lange unbeschadet überstehen können und wichtig für eine rasche Erholung der Mikroorganismen bei Wiedervernässung sind (Romani et al. 2013; Timoner et al. 2012). Insofern könnte die Enzymaktivität möglicherweise als Indikator für die Resistenz der mikrobiellen Gemeinschaften gegenüber Austrocknung dienen.

Auffallend an den Ergebnissen 2018 ist, dass in den feinkörnigen Sedimenten der burgenländischen und steirischen Bäche tendenziell die mittleren Enzymaktivitäten in den intermittierenden Abschnitten über jenen in den perennierenden lagen, während die mittleren Bakterienzahlen niedriger waren. In den Sedimenten des Kärntner Bachs zeigte sich das genau gegenteilige Muster. Ob es sich bei diesen Trends tatsächlich um signifikante belegbare Muster handelt bzw. welche Faktoren in diesem Fall hinter diesen Mustern liegen, ist ein Schwerpunkt der Untersuchungen 2019. Des Weiteren zeigen Studien, dass mikroskopische Algen auf Steinoberflächen leichter durch Austrocknung geschädigt werden als Bakterien (Acuna et al. 2015; Timoner et al. 2012). Da Algen eine wichtige Rolle in der Stoffaufnahme und im Stoffwechsel von Fließgewässern spielen, könnte sich eine Schädigung derselben zugunsten von Bakterien möglicherweise negativ auf die Selbstreinigungskapazität der betroffenen Bäche auswirken. Ein weiterer Schwerpunkt 2019 liegt daher auf algendominierten mikrobiellen Gemeinschaften von Steinoberflächen sowie auf jenen in tieferen Sedimentschichten (Hyporheal).

\section{Schlussfolgerungen und Ausblick}

Die Untersuchungen 2018 zeigen folgende vorläufige Tendenzen an: 
Tab. 1 Aktivität extrazellulärerEnzymeund Bakterienzahlen während der Trockenphase (Juli-September 2018) in den Sedimenten von perennierenden (per) und intermittierenden (int) Bächen im Burgenland (Bgld), der Steiermark (St) und Kärnten (K) (Mittelwerte und Standardabweichung; $n=5-15$ )

\begin{tabular}{|c|c|c|c|c|}
\hline Gewässertyp & $\begin{array}{l}\text { Glukosidase } \\
\mathrm{nmolg} \mathrm{g}^{-1} \mathrm{~h}^{-1}\end{array}$ & $\begin{array}{l}\text { Leucin-Aminopeptidase } \\
\mathrm{nmol} \mathrm{g}{ }^{-1} \mathrm{~h}^{-1}\end{array}$ & $\begin{array}{l}\text { Phosphatase } \\
\mathrm{nmolg}^{-1} \mathrm{~h}^{-1}\end{array}$ & $\begin{array}{l}\text { Bakterienzahlen } \\
10^{3} \text { Zellen } \mathrm{g}^{-1}\end{array}$ \\
\hline Bgld per & $19,5(11,1)$ & $55,3(38,5)$ & $13,5(4,9)$ & $7,7(3,9)$ \\
\hline Bgld int & $21,4(22,6)$ & $75,4(79,0)$ & $22,7(15,6)$ & $6,1(10,4)$ \\
\hline St per & $6,0(4,3)$ & $322,5(596,4)$ & $78,9(48,5)$ & $5,0(2,6)$ \\
\hline St int & $10,5(8,4)$ & $935,2(1264,1)$ & $123,8(111,7)$ & $4,1(3,1)$ \\
\hline $\mathrm{K}$ per & $11,6(3,3)$ & $248,7(21,5)$ & $12,4(2,9)$ & $15,5(4,5)$ \\
\hline $\mathrm{K}$ int & $8,3(1,1)$ & $224,8(51,1)$ & $7,8(3,7)$ & $18,5(10,4)$ \\
\hline
\end{tabular}

- Sowohl Beschattung durch Ufergehölz als auch ein hoher Anteil an Feinsedimenten scheinen die Wirkung von Austrocknung auf die mikrobielle Gemeinschaft in den Sedimenten reduzieren zu können.

- Langzeitige Auswirkungen auf die mikrobielle Gemeinschaft in den Sedimenten sind derzeit nicht feststellbar; allerdings können sich Veränderungen im Durchfluss in starken Schwankungen in den Nährstoffgehalten der Wassersäule auswirken, unabhängig davon, ob die Gewässer tatsächlich austrocknen oder nur eine deutlich reduzierte Wasserführung aufweisen.

Neben den angeführten Freilanduntersuchungen sind 2019 zwei Laborexperimente geplant, in denen Veränderungen der Stoffaufnahme und der mikrobiellen Gemeinschaften auf Steinoberflächen sowie im Sedimentkörper während der Austrocknung und der Wiedervernässung zeitlich detailliert und unter kontrollierten Bedingungen mittels experimenteller Rinnen und Sedimentreaktoren analysiert werden. Zusätzlich ist die Erstellung von 2D hydrodynamischen Modellen mit dem Softwarepaket RSim-2D (Tritthart et al. 2011) für drei ausgewählte Gewässerabschnitte im Gange (siehe Abb. 2), an denen Beprobungen durchgeführt wurden. Mit den Modellen werden die Benetzungszustände der Gewässersohle für derzeit auftretende charakteristische Abflüsse nachvollzogen und diese Information in einem weiteren Schritt mit den beobachteten biogeochemischen Parametern verschnitten. Durch Simulation der unter Klimawandelbedingungen zu erwartenden charakteristischen Abflüsse erlaubt das integrative Modell letztlich Rückschlüsse auf die zukünftige Selbstreinigungskapazität der Gewässer.

Darüber hinaus sollen im Rahmen eines Citizen Science Projekts auf Initiative der EU COST-Aktion SMIRES („Science and Management of Intermittent Rivers \& Ephemeral Streams"; www.smires.eu) intermittierende $\mathrm{Ge}$ wässer in Europa erstmalig möglichst flächig erfasst werden. Für die Meldung von intermittierenden Gewässern wird die von SPOTTERON für die Universität Zürich entwickelte und im Netz frei zur Verfügung gestellte App CrowdWater (https://crowdwater.ch/de/home) verwendet. Im Rahmen des Projekts PURIFY wurde eine Kooperation mit diesem CitizenScience-Projekt eingegangen, um möglichst umfassende Informationen über die Verbreitung von intermittierenden Gewässern in Österreich zu erhalten. Die Daten werden verwendet, um für das Projekt weitere geeignete Untersuchungsstellen $\mathrm{zu}$ identifizieren sowie um allgemein Informationen über die Verbreitung von intermittierenden Gewässern in Österreich zu erhalten.

Danksagung Dieses Projekt wird aus Mitteln des Klima- und Energiefonds gefördert und im Rahmen des Programms „Austrian Climate Research Programme ACRP 10“ durchgeführt
(ACRP10 - PURIFY - KR17AC0K13643). Das Projekt wird vom Amt der Kärntner Landesregierung, Abt. 8 (Umwelt, Wasser, Naturschutz), und vom Amt der Burgenländischen Landesregierung, Abt. 5 (Gewässeraufsicht), unterstützt. Die Freilanderhebungen und Laboranalysen wurden von Beate Pitzl, Annette Puritscher, Jakob Lechner, Aimee Jung und Michael Kolmar durchgeführt, die hydrodynamischen Modellierungen werden von Daniel Wildt bearbeitet. Wir danken unseren internationalen Partnern Daniel von Schiller, Universidad del País Vasco/Euskal Herriko Unibertsitatea (UPV-EHU), Spanien, und Michael Mutz, Brandenburgische Technische Universität Cottbus-Senftenberg, Deutschland, für ihre fachliche Expertise.

Funding Open access funding provided by University of Natural Resources and Life Sciences Vienna (BOKU).

Open Access Dieser Artikel wird unter der Creative Commons Namensnennung 4.0 International Lizenz (http:// creativecommons.org/licenses/by/4. 0/deed.de) veröffentlicht, welche die Nutzung, Vervielfältigung, Bearbeitung, Verbreitung und Wiedergabe in jeglichem Medium und Format erlaubt, sofern Sie den/die ursprünglichen $\mathrm{Au}$ tor(en) und die Quelle ordnungsgemäß nennen, einen Link zur Creative Commons Lizenz beifügen und angeben, ob Änderungen vorgenommen wurden. 
Acuna, V., Casellas, M., Corcoll, N., et al. (2015): Increasing extent of periods of no flow in intermittent waterways promotes heterotrophy. Freshwat Biol 60:1810-1823.

Bell, C.W., Fricks, B.E., Rocca, J.D., et al. (2013): High-throughput fluorometric measurement of potential soil extracellular enzyme activities. J Vis Exp (81). https://doi.org/10.3791/ 50961. Available via: http://www.jove.com/ video/50961. Accessed on 11 April 2019.

Bernal, S., Von Schiller, D., Sabater, F., Martí, E. (2013): Hydrological extremes modulate nutrient dynamics in mediterranean climate streams across different spatial scales. Hydrobiologia 719:31-42.

Datry, T., Bonada, N., Boulton, A.J. (2017) General introduction. In: Datry, T., Bonada, N., Boulton, A. (eds): Intermittent rivers and ephemeral streams: Ecology and Management. Academic Press, Elsevier, London. p. 1-20.

Datry, T., Larned, S.T., Tockner, K. (2014): Intermittent rivers: A challenge for freshwater ecology. Bioscience 64:229-235.

Delpla, I., Jung, A.V., Baures, E., et al. (2009): Impacts of climate change on surface water quality in relation to drinking water production. Environ Int 35:1225-1233.

Duhamel, S., Jacquet, S. (2006): Flow cytometric analysis of bacteria- and virus-like particles in lake sediments. J Microbiol Meth 64:316-332

Gomez, R., Arce, M.I., Baldwin D.S., Dahm, C.N. (2017): Water physicochemistry in intermittent rivers and ephemeral streams. In: Datry, T, Bonada, N., Boulton, A. (eds): Intermitten rivers and ephemeral streams: Ecology and $\mathrm{Ma}-$ nagement. Academic Press, Elsevier, London. p. 109-134

IPCC (2007): Contribution of Working Group II to the Fourth Assessment. Report of the Intergovernmental Panel on Climate Change. In Parry, M.L., Canziani, O.F, Palutikof, J.P., van der Linden, P.J., Hanson, C.E. (eds.): Climate Change
2007: Impacts, adaptation and vulnerability. Cambridge University Press, Cambridge, UK.

Marxsen, J., Zoppini, A., Wilczek, S. (2010): Microbial communities in streambed sediments recovering from desiccation. FEMS Microbiol Ecol 71:374-386.

McColl, R.H.S. (1974): Self-purification of small freshwater streams: phosphate, nitrate, and ammonia removal. New Zeal J Mar Fresh 8:375-388. Murdoch, P.S., Baron, J.S., Miller, T.L. (2000): Potential effects of climate change on surfacewater quality in North America. J Am Water Resour Assoc 36:347-366.

Oprei, A., Zlatanović, S., Mutz, M. (2019): Grazers superimpose humidity effect on stream biofilm resistance and resilience to dry-rewet stress. Sci Tot Environ 659:841-850.

Peterson, B.J., Wollheim, W.M., Mulholland, P.J., et al (2001): Control of nitrogen export from watersheds by headwater streams. Science 292:86-90.

Romani, A.M., Amalfitano, S., Artigas, J., et al. (2013): Microbial biofilm structure and organic matter use in mediterranean streams. Hydrobiologia 719:43-58.

Romani, A.M., Chauvet, E., Febria, C., et al. (2017): The biota of intermittent rivers and ephemeral streams: prokaryotes, fungi, and protozoans. In: Datry, T., Bonada, N., Boulton, A. (eds): Intermittent rivers and ephemeral streams: Ecology and Management. Academic Press, Elsevier London. 161-188.

Sabater, S., Timoner, X., Borrego, C., Acuña, V. (2016): Stream biofilm responses to flow intermittency: from cells to ecosystems. Front Environ Sci 4:1-10.

Von Schiller, D., Acuña, V., Graeber, D., et al (2011): Contraction, fragmentation and expansion dynamics determine nutrient availability in a Mediterranean forest stream. Aquat Sci 73:485-497.

Von Schiller, D., Bernal, S., Dahm, C.N., et a (2017): Nutrient and organic matter dynamics in intermittent rivers and ephemeral streams. In: Datry, T., Bonada, N., Boulton, A. (eds): Intermittent rivers and ephemeral streams: Ecology and Management. Academic Press, Elsevier, London. 135-160.

Sutherland, W.J., Bailey, M.J., Bainbridge, I.P., et al (2008): Future novel threats and opportunities facing UK biodiversity identified by horizon scanning. J Appl Ecol 45:821-833.

Timoner, X., Acuña, V., Von Schiller, D., Sabater, S. (2012): Functional responses of stream biofilms to flow cessation, desiccation and rewetting. Freshw Biol 57:1565-1578.

Tritthart, M., Liedermann, M., Schober, B., Habersack, H. (2011): Non-uniformity and layering in sediment transport modelling 2: river application. Journal of Hydraulic Research 49:335-344.

Weigelhofer, G., Hein, T., Bondar-Kunze, E. (2018): Phosphorus and nitrogen dynamics in riverine systems: Human impacts and management options. In: Schmutz, S., Sendzimir, J. (eds): Riverine ecosystem management: Science for governing towards a sustainable future. Aquatic Ecology Series Vol. 8. Springer, Cham. p. 187-202.

Whitehead, P.G., Wilby, R.L., Battarbee, R.W., et al (2009): A review of the potential impacts of climate change on surface water quality. Hydrol Sci J 54:101-123.

Wilby, R.L., Whitehead, P.G., Wade, A.J., et al (2006): Integrated modelling of climate change impacts on water resources and quality in a lowland catchment: River Kennet, UK. J Hydrol 330:204-220.

Hinweis des Verlags Der Verlag bleibt in Hinblick auf geografische Zuordnungen und Gebietsbezeichnungen in veröffentlichten Karten und Institutsadressen neutral. 